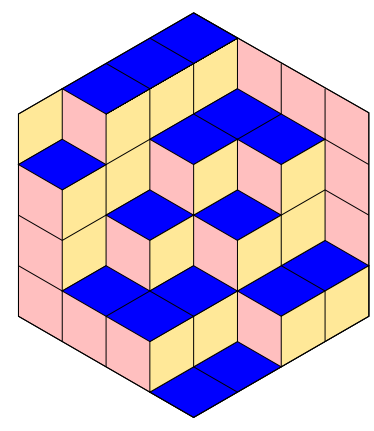

ALGEBRAIC COMBINATORICS

Fatemeh Mohammadi \& Kristin Shaw

Toric degenerations of Grassmannians from matching fields

Volume 2, issue 6 (2019), p. 1109-1124.

<http://alco.centre-mersenne.org/item/ALCO_2019__2_6_1109_0>

(C) The journal and the authors, 2019.

Some rights reserved.

(c) BY This article is licensed under the

Creative Commons ATtribution 4.0 InTERnational License.

http://creativecommons.org/licenses/by/4.0/

Access to articles published by the journal Algebraic Combinatorics on the website http://alco.centre-mersenne.org/ implies agreement with the Terms of Use (http://alco.centre-mersenne.org/legal/).

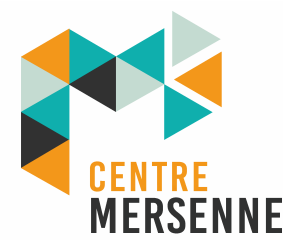

Algebraic Combinatorics is member of the Centre Mersenne for Open Scientific Publishing www.centre-mersenne.org 


\title{
Toric degenerations of Grassmannians from matching fields
}

\author{
Fatemeh Mohammadi \& Kristin Shaw
}

\begin{abstract}
We study the algebraic combinatorics of monomial degenerations of Plücker forms which is governed by matching fields in the sense of Sturmfels and Zelevinsky. We provide a necessary condition for a matching field to yield a SAGBI basis of the Plücker algebra for 3 -planes in $n$-space. When the ideal associated to the matching field is quadratically generated this condition is both necessary and sufficient. Finally, we describe a family of matching fields, called 2-block diagonal, whose ideals are quadratically generated. These matching fields produce a new family of toric degenerations of $\operatorname{Gr}(3, n)$.
\end{abstract}

\section{INTRODUCTION}

In this note we offer a new family of toric degenerations of $\operatorname{Gr}(3, n)$ arising from monomial degenerations of the Plücker forms. Toric degenerations provide a useful tool to study algebraic varieties. This is mainly because toric geometry is inextricably linked to the theory of polytopes and polyhedral fans. Combinatorial invariants of polytopes provide geometric information about toric varieties, and many of these invariants are preserved under degeneration. Here, a toric degeneration is a Gröbner degeneration such that the corresponding initial ideal is binomial and prime, see Definition 2.12 .

For general Grassmannians and flag varieties there are prototypic examples of toric degenerations which are related to Young tableaux, Gelfand-Cetlin integrable systems, and their polytopes $[1,17]$. In the case of the Grassmannian $\operatorname{Gr}(2, n)$, there are many other toric degenerations generalizing this primary example. Namely, any trivalent tree with $n$ number of labelled leaves gives rise to a toric degeneration of $\operatorname{Gr}(2, n)$. The toric variety is governed by the isomorphism type of the trivalent tree [25, 29]. These degenerations are related to bending systems on polygon spaces and integrable systems $[15,21]$.

The Gelfand-Cetlin degenerations arise from monomial initial degenerations of the Plücker forms. These degenerations arise from the general theory of Khovanskii bases, and in fact come from SAGBI bases for the Plücker algebra, see Definition 2.13. The leading term of each Plücker form in this case is the monomial of the determinant

Manuscript received 18th September 2018, revised 4th March 2019, accepted 21st March 2019.

KEYwORDS. toric degenerations, SAGBI and Khovanskii bases, Grassmannians, tropical geometry.

ACKNOWLEDGEMENTS. The first author's research was partially supported by the EPSRC Early Career Fellowship EP/R023379/1. The research of the second author was partially supported by the MPI Leipzig and the Bergen Research Foundation project grant "Algebraic and topological cycles in tropical and complex geometry". 
corresponding to the identity permutation. The new degenerations of $\operatorname{Gr}(3, n)$ provided here depend only on the underlying coherent matching field. A matching field is a choice of permutation for each Plücker form, see Definitions 2.2 and 2.8. A coherent matching field provides a monomial degeneration of the Plücker forms and are therefore candidates for SAGBI bases.

To a general matching field, we associate a toric ideal in the polynomial ring $\mathbb{K}\left[x_{i j}\right]$ where $\mathbb{K}$ is a field. These matching field ideals are most conveniently represented by matching field tableaux, which we also introduce here. These tableaux generalize Young tableaux which are usually strictly increasing in the columns. Columns of a matching field tableau are filled according to the permutation chosen by the matching field. A matching field ideal is the kernel of a monomial map, and hence toric, see Equation (2). Moreover, it is generated by binomials which come from pairs of matching field tableaux whose contents are row-wise equal. The Plücker forms are a SAGBI basis of the Plücker algebra with respect to a matching field if and only if its matching field ideal is equal to the initial ideal of the corresponding degeneration of the Plücker ideal, see Theorem 2.14 or [26, Theorem 11.4]. Therefore, obtaining a SAGBI basis from a matching field is equivalent to obtaining a toric degeneration of the Grassmannian.

From a weight matrix that produces a monomial degeneration of the Plücker forms, we can produce a tropical hyperplane arrangement, and the matching field can be determined from this geometric picture [10]. Using the associated tropical hyperplane arrangement, we introduce the notion of hexagonal matching fields of size $3 \times 6$ and non-hexagonal matching fields, see Definition 3.6. This leads to our first theorem.

THEOREM 1.1. If a $3 \times n$ matching field produces a toric degeneration of $\operatorname{Gr}(3, n)$, then it is non-hexgonal.

We also define submatching fields by using natural maps between Grassmannians of different sizes, see Definition 3.15. This allows us to extend Theorem 1.1 to higher Grassmannians.

THEOREM 1.2. A $k \times n$ matching field that has a hexagonal submatching field does not produce a toric degeneration of the Grassmannian $\operatorname{Gr}(k, n)$.

If a $3 \times n$ matching field ideal is quadratically generated, then the necessary condition from Theorem 1.1 is also sufficient.

THEOREM 1.3. A $3 \times n$ matching field whose ideal is quadratically generated provides a toric degeneration of $\operatorname{Gr}(3, n)$ if and only if it is non-hexagonal.

Describing a generating set of toric ideals is a well-studied and difficult problem. In particular, proving that an ideal is quadratically generated is quite a difficult task. There are some combinatorial criteria for the toric ideals arising from graphs, matroids and simplicial complexes to be generated by quadratics, see e.g. [28, $22,8,18]$. Such a criterion guarantees that the associated Koszul algebra is normal.

Not all coherent matching field ideals are quadratically generated. The first examples that we know of are of size $3 \times 8$. However, we introduce a class of matching fields of size $3 \times n$, called block diagonal, which are quadratically generated when they have two blocks.

THEOREM 1.4. The ideal of a 2-block diagonal matching field of size $3 \times n$ is quadratically generated.

COROLlary 1.5. A 2-block diagonal matching field produces a toric degeneration of the Grassmannian $\operatorname{Gr}(3, n)$. Equivalently, when $k=3$ the Plücker forms are a Khovanskii basis with respect to any weight matrix arising from a 2-block diagonal matching field. 
Before reviewing the contents of the paper we would like to comment on some related works. Rietsch and Williams describe families of toric degenerations of Grassmannians arising from plabic graphs [23]. In [4], Bossinger et. al. show that already in the case of $\operatorname{Gr}(3,6)$ there is a discrepancy between the toric degenerations arising on one hand from plabic graphs and on the other hand from top dimensional cones of the tropical Grassmannian. The toric degenerations studied here are a subset of the latter type coming from top dimensional cones of the tropical Grassmannian associated to Stiefel tropical linear spaces, see [10] for more details. In general, the combinatorial connection between matching fields and plabic graphs is still unknown. Kaveh and Manon provide a general connection between tropical geometry and Khovanskii bases (and hence toric degenerations) in [16]. This question has been studied in [5] for small flag varieties. Here, we are interested in determining when the Plücker forms are a SAGBI basis, equivalently when the associated initial degeneration of the Plücker ideal is toric. The results presented here offer a family of examples that fit into the general framework of Kaveh and Manon and which are linked to combinatorics. Lastly, we would like to remark that toric degenerations of flag varieties and Schubert varieties arising from matching fields is another open direction of research at the present time.

We finish the introduction with an outline of the paper. Section 2 fixes notations for the Grassmannians and introduces matching fields. In Section 3, we review the connection between matching fields and tropical hyperplane arrangements. Here we introduce the notion of hexagonal matching fields and prove Theorems 1.1, 1.2, and 1.3. Block diagonal matching fields are introduced in Section 4 and the proof of Theorem 1.4 is also given here. The final section defines matching field polytopes and provides some examples as well as remarks about their combinatorics.

\section{Preliminaries}

Throughout we set $[n]:=\{1, \ldots, n\}$ and we use $\mathbf{I}_{k, n}$ to denote the collection of subsets of $[n]$ of size $k$. The symmetric group on $k$ elements is denoted by $S_{k}$. We also fix a field $\mathbb{K}$.

The Grassmannian $\operatorname{Gr}(k, n)$ is the space of all $k$ dimensional linear subspaces of $\mathbb{K}^{n}$. A point in $\operatorname{Gr}(k, n)$ can be represented by a $k \times n$ matrix with entries in $\mathbb{K}$. Let $X=\left(x_{i j}\right)$ be a $k \times n$ matrix of indeterminates. For a subset $I=\left\{i_{1}, \ldots, i_{k}\right\} \in \mathbf{I}_{k, n}$, let $X_{I}$ denote the $k \times k$ submatrix with the column indices $i_{1}, \ldots, i_{k}$. The Plücker forms (or Plücker coordinates) are $P_{I}=\operatorname{det}\left(X_{I}\right)$ for $I \in \mathbf{I}_{k, n}$. These forms determine the Plücker embedding from $\operatorname{Gr}(k, n)$ into $\mathbb{P}\left(\begin{array}{l}n \\ k\end{array}\right)-1$.

In the following, we consider the polynomial ring $\mathbb{K}\left[x_{i j}\right]$ on the variables $x_{i j}$ with $1 \leqslant i \leqslant k$ and $1 \leqslant j \leqslant n$ and the polynomial ring $\mathbb{K}\left[P_{I}\right]$ on the Plücker variables with $|I|=k$.

Definition 2.1. The Plücker ideal $\mathcal{I}_{k, n}$ is defined as the kernel of the map

$$
\begin{aligned}
\psi: \mathbb{K}\left[P_{I}\right] & \rightarrow \mathbb{K}\left[x_{i j}\right] \\
P_{I} & \mapsto \operatorname{det}\left(X_{I}\right) .
\end{aligned}
$$

The Plücker algebra is the finitely generated algebra $\mathbb{K}\left[P_{I}\right] / \mathcal{I}_{k, n}$ denoted by $\mathcal{A}_{k, n}$.

Definition 2.2. A $k \times n$ matching field is a map $\Lambda: \mathbf{I}_{k, n} \rightarrow S_{k}$.

Given a $k \times n$ matching field $\Lambda$ and a subset $I=\left\{i_{1}, \ldots, i_{k}\right\} \in \mathbf{I}_{k, n}$ we consider the set to be ordered by $i_{1}<\cdots<i_{k}$. We think of the permutation $\sigma=\Lambda(I)$ as inducing a new ordering on the elements of $I$ where the position of $i_{s}$ is determined by the value of $\sigma(s)$. It is convenient to represent the variable $P_{I}$ as a $k \times 1$ tableau where $(\sigma(r), 1)$ contains $i_{r}$. 
Definition 2.3. Let $\Lambda$ be a size $k \times n$ matching field. A $\Lambda$-tableau is a tableau of size $k \times d$ for any $d \geqslant 1$ with entries in $[n]$, so that the entries in each column are pairwise distinct and filled according to the order determined by $\Lambda$.

EXAMPLE 2.4. The diagonal matching field assigns to each subset $I \in \mathbf{I}_{k, n}$ the identity permutation [27, Example 1.3]. Therefore, a $\Lambda$-tableau is a rectangular tableau of size $k \times d$ filled with entries in $[n]$ such that the columns are strictly increasing.

EXAmPle 2.5. A $k \times n$ matching field is called pointed if there exists $i_{1}, \ldots, i_{k} \in[n]$ such that if $i_{s} \in I$ for some $1 \leqslant s \leqslant k$ then $\Lambda(I)(s)=s$ [27, Example 1.4]. In other words, if $i_{s} \in I$ for some $1 \leqslant s \leqslant k$ then the column corresponding to $P_{I}$ contains $i_{s}$ in row $s$. Below are the tableaux representing $P_{I}$ for a pointed matching field of size $3 \times 5$ which is otherwise filled diagonally:

$\begin{array}{llllllll}1 & 1 & 1 & 1 & 1 & 4 & 5 & 4 \\ 2, & 2, & 2, & 4, & 5, & 2, & 2, & 2 \\ 3 & 4 & 5 & 3 & 3 & 3 & 3 & 5\end{array}$.

Generalising this, we say that a size $k \times n$ matching field $\Lambda$ is pointed on $S \subset[n]$ if for all $i \in S$ there exists a $j_{i}$ such that $i$ always appears in row $j_{i}$ of any $\Lambda$ matching field tableau. Here $S$ need not have size equal to $k$.

A monomial $\Pi_{I \in A} P_{I}$ can be represented by a $\Lambda$-tableau of size $k \times|A|$ given by the concatenation of the columns with content $I$ filled according to the matching field. To each monomial $\Pi_{I \in A} P_{I}$ we associate a sign $\epsilon_{A}= \pm 1$ determined by the signature of the permutations $\Lambda(I)$ for all $I \in A$. More precisely,

$$
\epsilon_{A}=\Pi_{I \in A} \operatorname{sgn}(\Lambda(I)) .
$$

Definition 2.6. Given a matching field $\Lambda$, the matching field ideal $\mathcal{I}_{\Lambda} \subset \mathbb{K}\left[x_{i j}\right]$ is generated by the binomial relations

$$
\epsilon_{A} \Pi_{I \in A} P_{I}-\epsilon_{B} \Pi_{J \in B} P_{J}
$$

if and only if the contents of the corresponding $\Lambda$-tableau of size $k \times|A|$ are row-wise equal.

To $I \in \mathbf{I}_{k, n}$ with $\sigma=\Lambda(I)$ we associate the monomial

$$
\mathbf{x}_{\Lambda(I)}:=x_{\sigma(1) i_{1}} x_{\sigma(2) i_{2}} \cdots x_{\sigma(k) i_{k}} .
$$

A $k \times n$ matching field $\Lambda$, gives a map of polynomial rings

$$
\begin{aligned}
\phi_{\Lambda}: \mathbb{K}\left[P_{I}\right] & \rightarrow \mathbb{K}\left[x_{i j}\right] \\
P_{I} & \mapsto \operatorname{sgn}(\Lambda(I)) \mathbf{x}_{\Lambda(I)} .
\end{aligned}
$$

Proposition 2.7. Given a matching field $\Lambda$, the matching field ideal $\mathcal{I}_{\Lambda}$ is the kernel of the monomial map $\phi_{\Lambda}$ from Equation (3).

Definition 2.8. A $k \times n$ matching field $\Lambda$ is coherent if there exists a $k \times n$ matrix $M$ with entries in $\mathbb{R}$ such that for every $I \in \mathbf{I}_{k, n}$ the initial form of the Plücker form $P_{I} \in \mathbb{K}\left[x_{i j}\right]$, the sum of all terms in $M_{I}$ of lowest weight, is $\operatorname{in}_{M}\left(P_{I}\right)=\operatorname{sgn}(\Lambda(I)) \mathbf{x}_{\Lambda(I)}$. In this case, we say that the matrix $M$ induces the matching field $\Lambda$.

EXAMPLE 2.9. When $k=2$ all coherent matching fields are induced by a total ordering on the set $[n]$, see [27, Proposition 1.11]. 
EXAMPLE 2.10. The diagonal matching field of size $k \times n$ is coherent [27, Example 1.3]. For example, this matching field is induced by a $k \times n$ weight matrix $M$ whose $i, j$-th entry is $(i-1)(n-j)$. Therefore, we have,

$$
M=\left[\begin{array}{ccccc}
0 & 0 & 0 & \cdots & 0 \\
n-1 & \cdots & 2 & 1 & 0 \\
2(n-1) & \cdots & 4 & 2 & 0 \\
\cdots & \cdots & \cdots & \cdots & \\
(k-1)(n-1) & \cdots & 2(k-1) & k-1 & 0
\end{array}\right] .
$$

For any size $k$ subset $I=\left\{i_{1}, i_{2}, \ldots, i_{k}\right\}$ with $i_{1}<i_{2}<\cdots<i_{k}$ the unique term in the determinant of $M_{I}$ with the lowest weight is the diagonal term.

EXAMPLE 2.11. Recall the notion of a pointed matching field from Example 2.5. If a pointed matching field is coherent then it can be induced by a weight matrix $M$ such that for all $1 \leqslant s \leqslant k$ the $i_{s}$-th column has entries $M \gg 0$ except for in row $s$ where the entry can be chosen to be 0 .

DEFINITION 2.12. Let $\mathcal{I}$ be an ideal in the polynomial ring $S=\mathbb{K}\left[y_{1}, \ldots, y_{m}\right]$ and let $w \in \mathbb{R}^{m}$. The initial degeneration with respect to $w$ is called toric if the initial ideal $\operatorname{in}_{w}(\mathcal{I})$ is prime and binomial.

Definition 2.13 ([24]). The set of Plücker forms $\left\{P_{I}\right\}_{I \in \mathbf{I}_{k, n}} \subset \mathbb{K}\left[x_{i j}\right]$ is a SAGBI basis for the Plücker algebra $\mathcal{A}_{k, n}$ with respect to a weight matrix $M$ if for all $I \in \mathbf{I}_{k, n}$ the initial form $\operatorname{in}_{M}\left(P_{I}\right)$ is a monomial and $\operatorname{in}_{w_{M}}\left(\mathcal{A}_{k, n}\right)=\mathbb{K}\left[\operatorname{in}_{M}\left(P_{I}\right)\right]_{I \in \mathbf{I}_{k, n}}$. Here $w_{M}$ is the weight vector on the variables $P_{I}$ induced by the weight matrix $M \in \mathbb{R}^{k \times n}$ on the variables $x_{i j}$.

The following theorem intimately relates SAGBI bases and toric initial degenerations. It is phrased in the context of matching fields and Grassmannians.

THEOREM 2.14 ([26, Theorem 11.4]). The set of Plücker forms $\left\{P_{I}\right\}_{I \in \mathbf{I}_{k, n}} \subset \mathbb{K}\left[x_{i j}\right]$ is a SAGBI basis with respect to a weight matrix $M$ if and only if $\operatorname{in}_{w_{M}}\left(\mathcal{I}_{k, n}\right)=\mathcal{I}_{\Lambda}$, where $w_{M}$ is the weight vector on the variables $P_{I}$ induced by $M$ and $\Lambda$ is the matching field induced by $M$.

\section{CohEREnt MATCHING FiELDS AND TROPICAL HYPERPLANE ARRANGEMENTS}

In [10], Fink and Rincón provide a link between tropical hyperplane arrangements and coherent matching fields (and more generally multi-matching fields). We will summarize the facts needed here and refer the reader to [10] for more details. In [10], tropical hyperplane arrangements are described in tropical projective space. We do not require this level of generality here, therefore we simplify our considerations in the following summary.

Let $M=\left(a_{i j}\right) \in \mathbb{R}^{k \times n}$ be a weight matrix. For each $1 \leqslant j \leqslant n$ consider the piecewise linear function $F_{j}: \mathbb{R}^{k-1} \rightarrow \mathbb{R}$, given by

$$
F_{j}(x)=\max \left\{a_{1 j}, a_{2 j}+x_{2}, \ldots, a_{k j}+x_{k}\right\} .
$$

In $\mathbb{R}^{k-1}$ let $\Sigma$ be the $k-2$ dimensional polyhedral fan whose top dimensional cones are spanned by subsets of size $k-2$ of the vectors $v_{1}, \ldots, v_{k}$, where $v_{1}=$ $(1, \ldots, 1)$ and $v_{i}=-e_{i-1}$ otherwise. For $k=3$ this amounts to three rays in the directions $(1,1),(-1,0)$ and $(0,-1)$ emanating from the origin. If $a_{1 j}=0$, then the non-differentiability locus of $F_{j}$ is the fan $\Sigma \subset \mathbb{R}^{k-1}$ translated by the vector $\left(-a_{2 j}, \ldots,-a_{k j}\right) \in \mathbb{R}^{k-1}$. Any coherent matching field is induced by a weight matrix $M$ whose first row consists of zeros. So we may assume that $a_{1 j}=0$ for all $j$. 
REMARK 3.1. In this paper we purposely use the minimum conventions for tropical arithmetic and the maximum conventions for tropical geometry. We do this to avoid the appearance of many minus signs when passing from the algebra of weight matrices to the geometry of tropical hyperplane arrangements.
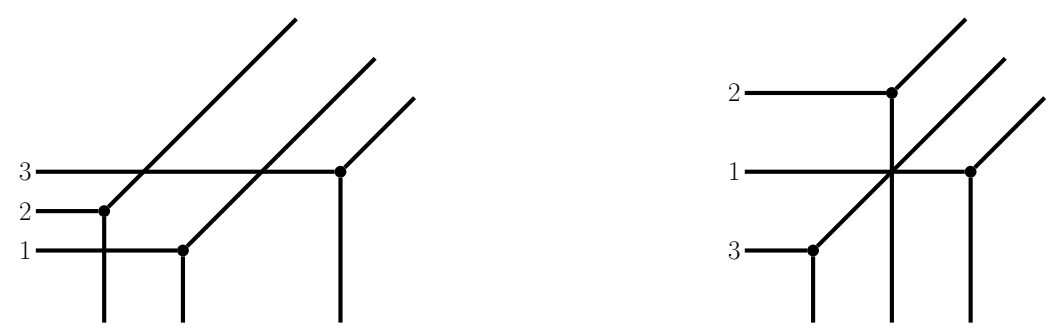

FiguRE 1. Two arrangements of three tropical lines in $\mathbb{R}^{2}$. On the left the configuration intersects properly on the right the three lines are concurrent.

A $k \times n$ weight matrix $M \in \mathbb{R}^{k \times n}$, whose first row consists of zeros, produces an arrangement of tropical hyperplanes $\mathcal{A}=\left\{H_{1}, \ldots, H_{n}\right\}$ defined by the functions $F_{1}, \ldots, F_{n}$, whose coefficients come from $M$. To each $k-1$ dimensional cell $\tau$ of the complement of the arrangement $\mathcal{A}$ in $\mathbb{R}^{k-1}$ there is an associated covector $c_{\tau} \in \mathcal{P}[n]^{k}$, where $\mathcal{P}[n]$ denotes the power set of $n$. The $i$-th entry of the covector $c_{\tau}$ is a subset $S_{i} \subset[n]$ corresponding to the collection of hyperplanes in $\mathcal{A}$ which intersect the ray $x+t v_{i}$ for $x \in \tau^{\circ}$ and $t \geqslant 0$. Here the vectors $v_{i}=-e_{i-1}$ for $i=2, \ldots, k-1$ and $v_{1}=(1, \ldots, 1)$. The coarse covector of a cell is simply the vector which records the sizes of the subsets of the covector.

Definition 3.2. A collection of $k$ hyperplanes $H_{1}, \ldots, H_{k}$ in $\mathbb{R}^{k-1}$ is said to intersect properly if $\cap_{i=1}^{k} H_{i}=\varnothing$. Equivalently, a collection of $k$ hyperplanes $H_{1}, \ldots, H_{k}$ in $\mathbb{R}^{k-1}$ intersects properly if and only if there is a $k-1$ dimensional cell in the complement of $\cup_{i=1}^{k} H_{i}$ whose coarse covector is $(1, \ldots, 1)$.

EXAMPLE 3.3. Consider the two $3 \times 3$ weight matrices,

$$
M_{1}=\left[\begin{array}{lll}
0 & 0 & 0 \\
4 & 2 & 8 \\
2 & 3 & 4
\end{array}\right], \quad M_{2}=\left[\begin{array}{lll}
0 & 0 & 0 \\
6 & 4 & 2 \\
4 & 6 & 2
\end{array}\right] .
$$

The matrix $M_{1}$ corresponds to tropical lines intersecting properly in Figure 1 (left) and the matrix $M_{2}$ corresponds to concurrent tropical lines in Figure 1 (right).

The following proposition can be extracted from [7, Proposition 2.4] and [10, Propositions 5.11 and 5.12].

Proposition 3.4. Let $M$ be a $k \times n$ weight matrix such that for any size $k$ subset $I \subset[n]$, the collection of hyperplanes $\left\{H_{i}\right\}_{i \in I}$ intersects properly. Then

$$
\operatorname{in}_{M}\left(P_{I}\right)=\operatorname{sgn}(\Lambda(I)) x_{1 c_{1}} x_{2 c_{2}} \ldots x_{k c_{k}}
$$

where $\left(c_{1}, \ldots, c_{k}\right)$ is the covector of the unique cell with coarse covector $(1,1, \ldots, 1)$ and $\operatorname{sgn}(\Lambda(I))$ is the sign of the permutation $i \mapsto \sigma(i)$, where $c_{\sigma(1)}<\cdots<c_{\sigma(k)}$.

COROllary 3.5. A $k \times n$ weight matrix provides a monomial degeneration of the Plücker forms if and only if for any size $k$ subset $I \subset[n]$ the collection of tropical hyperplanes $\left\{H_{i}\right\}_{i \in I}$ intersects properly. 
Next we compare the matching field ideal $\mathcal{I}_{\Lambda}$ with the initial degeneration of the Plücker ideal $\mathcal{I}_{k, n}$ with respect to the weights on the Plücker forms induced by $\Lambda$.

Definition 3.6. A coherent matching field $\Lambda: \mathbf{I}_{3,6} \rightarrow S_{3}$ is hexagonal if it is the matching field of a tropical hyperplane arrangement whose unique cell with coarse covector $(2,2,2)$ is a hexagon. A matching field $\Lambda: \mathbf{I}_{3, n} \rightarrow S_{3}$ is non-hexagonal if for every size six subset $J$ the matching field $\left.\Lambda\right|_{J}$ is not hexagonal.

For a homogeneous ideal $\mathcal{I}$ we let $\mathcal{I}_{d}$ denote the elements of degree $d$.

Proposition 3.7. Let $M \in \mathbb{R}^{3 \times n}$ be a weight matrix such that $\operatorname{in}_{M}\left(P_{I}\right)$ is a monomial for all Plücker forms $P_{I}$. Then $\left(\mathcal{I}_{\Lambda}\right)_{2}=\left(\operatorname{in}_{w_{M}}\left(\mathcal{I}_{3, n}\right)\right)_{2}$ if and only if the matching field is non-hexagonal.

Before giving the proof we pause to illustrate the condition presented in the above proposition with two examples.
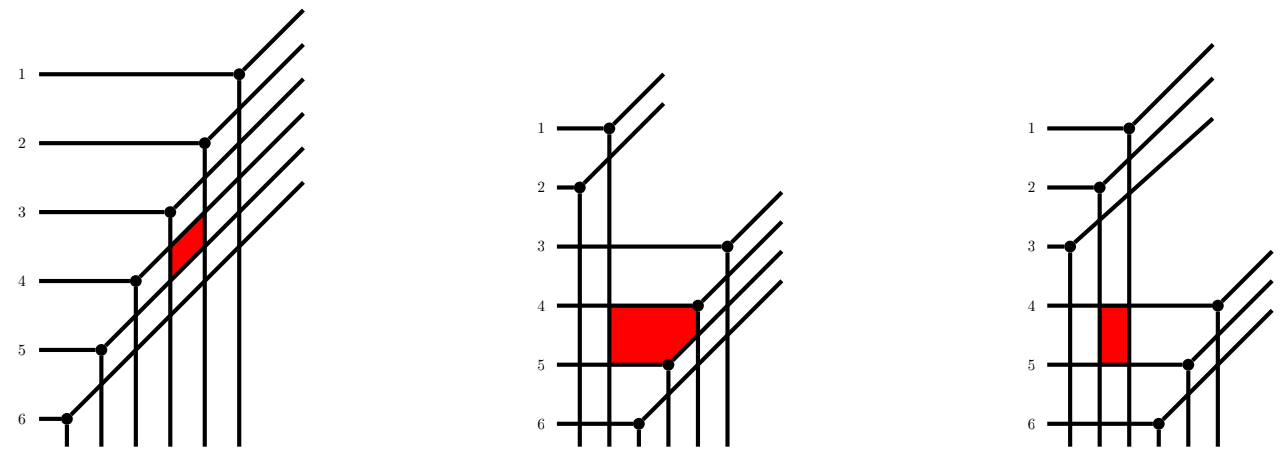

FiguRE 2. On the left a tropical hyperplane arrangement yielding the diagonal matching field of size $3 \times 6$, in the middle an arrangement yielding the block diagonal matching field $B \Lambda_{2,4}$ and on the right a block diagonal matching field $B \Lambda_{3,3}$. See Section 4 for the definition of a block diagonal matching field. In each arrangement, the $(2,2,2)$ cell is shaded in red.

EXAMPLE 3.8 (Diagonal matching field). The associated hyperplane arrangement of the following weight matrix is depicted in Figure 2 (left).

$$
M=\left[\begin{array}{cccccc}
0 & 0 & 0 & 0 & 0 & 0 \\
6 & 5 & 4 & 3 & 2 & 1 \\
11 & 9 & 7 & 5 & 3 & 1
\end{array}\right]
$$

The initial terms are $x_{1 i} x_{2 j} x_{3 k}$ for $1 \leqslant i<j<k \leqslant 6$. The following $\Lambda$-tableaux indicate the row-wise equal tableaux which give all the binomial relations in $\mathcal{I}_{\Lambda}$ of multi-degree $e_{J}$ for $|J|=6$,

$$
\begin{aligned}
& \begin{array}{llllllll}
12 & 12 & 12 & 12 & 13 & 13 & 12 & 12
\end{array} \\
& 34=34=43=43, \quad 24=24, \quad 35=53 \\
& \begin{array}{llllllll}
56 & 65 & 56 & 65 & 56 & 65 & 46 & 64
\end{array}
\end{aligned}
$$

Notice that in each of the equivalence classes of the quadratic monomials listed above the first monomial listed is a semi-standard tableaux, i.e. all rows are in weakly increasing order and the columns are strictly increasing and it is the only semistandard tableaux of that equivalence class. The other quadratic terms of multi-degree 
$(1, \ldots, 1)$ are

14
25
36 $\quad$ and $\quad \begin{aligned} & 13 \\ & 25 \\ & 46\end{aligned}$

Notice that they are independent in $\mathbb{K}\left[P_{I}\right]$.
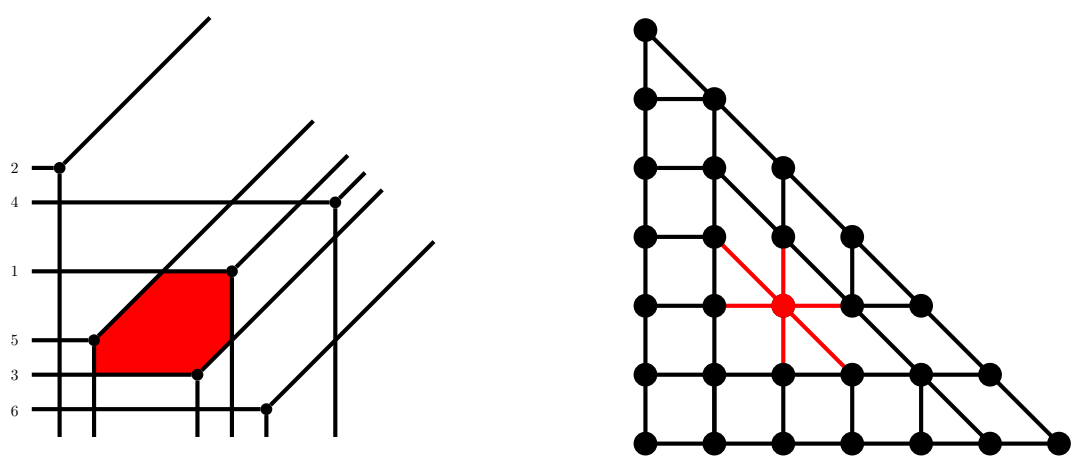

FigURE 3. The tropical hyperplane arrangement from the weight matrix in Example 3.9 on the left and its dual regular subdivision on the 2-dimensional simplex of size six. The region shaded in red is the $(2,2,2)$-cell and is bounded by the lines $L_{1}, L_{5}$, and $L_{3}$.

EXAMPLE 3.9 (Hexagonal matching field). The associated hyperplane arrangement of the following weight matrix

$$
M=\left[\begin{array}{llllll}
0 & 0 & 0 & 0 & 0 & 0 \\
6 & 1 & 5 & 9 & 2 & 7 \\
5 & 8 & 2 & 7 & 3 & 1
\end{array}\right]
$$

is depicted in Figure 3 (left). The initial terms of the Plücker forms are:

$123,421,125,126,413,153,136,451,416,156,423,523,326,425,426$,

$526,453,436,356$, and 456 ,

where by $i j k$ we mean $x_{1 i} x_{2 j} x_{3 k}$. The following $\Lambda$-tableaux indicate the row-wise equal tableaux which give all the binomial relations in $\mathcal{I}_{\Lambda}$ of the form $e_{J}$ for $|J|=6$.

$$
\begin{aligned}
& 14 \quad 41 \quad 14 \quad 14 \quad 54 \quad 54 \\
& 25=25=52=25, \quad 21=21 \text {, } \\
& \begin{array}{llllll}
36 & 36 & 36 & 63 & 36 & 63
\end{array} \\
& 41 \quad 41 \quad 43 \quad 43 \\
& 23=32, \quad 25=52 \text {. } \\
& 56 \quad 65 \quad 16 \quad 16
\end{aligned}
$$

Notice that compared with Example 3.8, there is an additional binomial relation listed.

EXAMPLE 3.10. The matching field ideal in Example 3.8 is generated by 35 binomials and it is equal to the initial ideal of $\mathcal{I}_{3, n}$. However, in Example 3.9, the ideal $\mathcal{I}_{\Lambda}$ is generated by 36 binomials. More precisely, the relation $P_{523} P_{416}-P_{526} P_{413}$ is in $\mathcal{I}_{\Lambda}$, but not in the initial ideal $\operatorname{in}_{w_{M}}\left(\mathcal{I}_{3, n}\right)$. 
LEMMA 3.11. Let $M$ be a $3 \times n$ weight matrix providing a monomial degeneration of the Plücker forms. Then for any size six subset $J \subset[n]$, the $(2,2,2)$-cell of $\left.\mathcal{A}\right|_{J}$ determines the initial terms of exactly eight Plücker forms. Moreover, these eight initial terms come in four pairs which produce quadratic relations in $\mathcal{I}_{\Lambda}$.

Proof. Suppose that the covector of the $(2,2,2)$-cell is $\left(S_{1}, S_{2}, S_{3}\right)$ where $\left|S_{i}\right|=2$ for all $i$. Then choosing $s_{i} \in S_{i}$ for $i=1,2,3$ we obtain $\operatorname{in}_{M}\left(P_{s_{1} s_{2} s_{3}}\right)= \pm x_{1 s_{1}} x_{2 s_{2}} x_{3 s_{3}}$ by Proposition 3.4. Therefore the first claim follows.

For simplicity we can assume that $J=\{1, \ldots, 6\}$ and that

$$
\left(S_{1}, S_{2}, S_{3}\right)=(\{1,4\},\{2,5\},\{3,6\}),
$$

as in the case for the hexagon cell in Figure 3 . Then the following $\Lambda$-tableaux are all row-wise equal and give rise to three binomial relations in $\mathcal{I}_{\Lambda}$,

$$
\begin{aligned}
& 14 \quad 41 \quad 14 \quad 14 \\
& 25=25=52=25 \text {. } \\
& 36 \quad 36 \quad 36 \quad 63
\end{aligned}
$$

This completes the proof.

Proof of Proposition 3.7. Suppose we have an arrangement of 6 tropical lines in $\mathbb{R}^{2}$. The cell which has coarse covector $(2,2,2)$ is a hexagon if and only if the edges with endpoints $(2,2,2)-(\sigma(1), \sigma(2), \sigma(3))$ for all $\sigma \in S_{3}$ are present in the dual subdivision of $6 \Delta_{2}$. See the right hand side of Figure 3 .

Suppose without loss of generality that the covector of the hexagon cell is $(\{1,4\},\{2,5\},\{3,6\})$, as it is for example in Figure 3. If the cell dual to $(2,2,2)$ is a hexagon, then up to the appropriate labeling there are covectors,

$$
(\{1,4\},\{2\},\{3,5,6\}) \quad \text { and } \quad(\{1,4\},\{2,3,5\},\{6\}) .
$$

From this pair of covectors we obtain the quadratic relation

$$
\begin{aligned}
& 14 \\
& 23=23 \\
& 56 \\
& 56
\end{aligned}
$$

in the ideal $\mathcal{I}_{\Lambda}$.

There are four other lattice points that are endpoints of the six segments of the subdivision dual to the hexagon. They come in two pairs formed by the points which are on the same line. For each of these pairs we obtain a new independent quadratic relation in $\mathcal{I}_{\Lambda}$ as above.

Taking into account the quadratic relations from Lemma 3.11 as well, we can conclude that the dimension of the $e_{1}+\cdots+e_{6}$ graded piece of the coordinate ring of the toric variety $\mathcal{I}_{\Lambda}$ is at most four. However, the dimension of this piece for the coordinate ring of $\mathcal{I}_{3, n}$ and hence also of $\operatorname{in}_{w_{M}}\left(\mathcal{I}_{3, n}\right)$ is five. This dimension is given by the number of semi-standard tableaux with content $\{1,2,3,4,5,6\}$ of size $3 \times 2$.

For the other direction, we again consider the multi-grading on the coordinate ring of $I_{\Lambda}$. The degree two part of this coordinate ring has elements which have two types with respect to the multi-grading. They are either $e_{J}$ or $e_{J}+e_{i}-e_{j}$ for $i, j \in J$ and $i \neq j$ where $J$ is a size six subset. The dimension of the $e_{J}+e_{i}-e_{j}$ piece of the coordinate ring is of the correct dimension for any $J$ and $i, j$. This follows from $[25$, Corollary 4.4].

Finally, we consider the terms with $e_{J}$ multi-grading for a $J$ with $|J|=6$. If $\Lambda$ is not hexagonal, then the dual subdivision of a tropical hyperplane arrangement inducing $\Lambda$ must be missing one of the possible edges with endpoint $(2,2) \in 6 \Delta$. Proceeding case by case, we can verify the statement of the proposition. 
Proof of Theorem 1.3. Assume that $\mathcal{I}_{\Lambda}$ is quadratically generated. If the matching field $\Lambda$ is hexagonal, then by Proposition 3.7 there is a size six subset $J \subset[n]$ such that $\left(\mathcal{I}_{\Lambda}\right)_{2} \neq\left(\mathrm{in}_{w_{M}}\left(\mathcal{I}_{3, n}\right)\right)_{2}$. However, by [26, Theorem 11.4], equality of the ideals $\mathcal{I}_{\Lambda}$ and $\operatorname{in}_{w_{M}}\left(\mathcal{I}_{3, n}\right)$ is a necessary and sufficient condition for the Plücker forms to be a SAGBI basis. This proves one direction.

For the other direction, we compare the ideals $\operatorname{in}_{w_{M}}(\mathcal{I})$ and $\mathcal{I}_{\Lambda}$, and then we complete the proof by applying Theorem 2.14. Since $\mathcal{I}_{\Lambda}$ is quadratically generated, there are two types of generators determined by their multi-degrees. There are generators of type $e_{J}$ or of type $e_{J}+e_{i}-e_{j}$ where $|J|=6$ and $i, j \in J$. We show that the generators of $\mathcal{I}_{\Lambda}$ are included in $\left(\mathrm{in}_{w_{M}}\left(\mathcal{I}_{3, n}\right)\right)_{2}$ by considering each type.

Firstly, the generators of type $e_{J}+e_{i}-e_{j}$ can be reduced to the case of $\operatorname{Gr}(2,5)$. In this case the statement holds since the Plücker forms are a SAGBI basis with respect to any coherent matching field $\Lambda^{\prime}: \mathbf{I}_{2,5} \rightarrow S_{2}[25]$.

For generators of type $e_{J}$ where $|J|=6$, we reduce to the situation of $\operatorname{Gr}(3,6)$ and matching fields of the form $\Lambda^{\prime}: \mathbf{I}_{3,6} \rightarrow S_{3}$. Combining Proposition 3.7 and [26, Theorem 11.4] shows that the Plücker forms are a SAGBI basis with respect to any of these coherent matching fields. Therefore the subduction algorithm terminates with a constant for any generator of type $e_{J}$ when the restriction to $J$ is not hexagonal. By again applying [26, Corollary 11.5] we prove the other direction and our theorem.

Following Theorem 1.3, we are interested in determining when a $3 \times n$ matching field ideal is quadratically generated.

EXAMPLE 3.12. The ideal of the diagonal matching field from Example 2.4 is quadratically generated, see [20, Theorem 14.16].

EXAmPLE 3.13. Consider the $2 \times 6$ matching field $\Lambda$ that assigns the transposition (12) for sets $I \in\{\{1,4\},\{2,3\},\{3,6\},\{4,5\}\}$ and the identity permutation otherwise. A minimal generator of the matching field ideal is

$$
\begin{aligned}
& 135 \\
& 246
\end{aligned}=\begin{aligned}
& 135 \\
& 624
\end{aligned} .
$$

Therefore, this matching field ideal is not quadratically generated.

REMARK 3.14. The matching field of the non-quadratically generated ideal in Example 3.13 is not coherent since it does not arise from a total ordering on the set $[n]$. Our smallest known examples of coherent matching fields whose ideals are not quadratically generated are of size $3 \times 8$ and were found via a random search. In our random checks, approximately $0.03 \%$ of coherent matching fields of size $3 \times 8$ produced ideals which were not quadratically generated. A random search of coherent matching fields of size $3 \times 7$ produced no non-quadratically generated matching field ideals.

Before presenting the proof of Theorem 1.2 we introduce the notion of submatching field. Before defining hexagonal submatching fields, recall the notion of a matching field being pointed on a subset $S$ of $[n]$ from Example 2.5.

DeFinition 3.15. Given a matching field $\Lambda$ and two subsets $S \subset T \subset[n]$, the submatching field $\left.\Lambda\right|_{T-S, T}$ of $\Lambda$ is obtained by restricting $\Lambda$ to subsets $I$ of $[n]$ with $S \subset I \subset T$ and restricting the matching to $I \backslash S$.

The submatching field $\left.\Lambda\right|_{T-S, T}$ is hexagonal if it is a size $3 \times 6$ hexagonal matching field and $\Lambda$ is pointed on $S \subset[n]$.

Proof of Theorem 1.2. Let $\left.\Lambda\right|_{T-S, T}$ be a hexagonal submatching field of $\Lambda$. Consider the graded piece of the Plücker algebra consisting of degree two monomials in the variables $P_{I}$ such that $S \subset I \subset T$. This vector space has the same dimension as the 
degree two graded piece of $\mathcal{A}_{3,6}$, and this is five dimensional. However, the analogous graded piece of $\mathbb{K}\left[x_{i j}\right] / \mathcal{I}_{\Lambda}$ is only four dimensional since $\mathcal{I}_{\Lambda}$ consists of the list of binomials from Example 3.9. The graded Hilbert functions of $\mathcal{A}_{k, n}$ and $\mathbb{K}\left[x_{i j}\right] / \mathcal{I}_{\Lambda}$ are not equal and therefore the matching field $\Lambda$ does not produce a toric degeneration of $\operatorname{Gr}(k, n)$.

\section{BLOCK DIAGONAL MATCHING FIELDS}

In this section we describe a family of coherent matching fields of size $3 \times n$ whose toric ideals are generated in degree two and therefore yield toric degenerations and SAGBI bases of $\operatorname{Gr}(3, n)$.

Consider a sequence of positive numbers $a_{1}, a_{2}, \ldots, a_{r}$ so that $\sum_{i=1}^{r} a_{i}=n$. For $1 \leqslant s \leqslant r$ set $I_{s}=\left\{\alpha_{s-1}+1, \alpha_{s-1}+2, \ldots, \alpha_{s}\right\}$, where $\alpha_{s}=\sum_{i=1}^{s} a_{i}$ and $\alpha_{0}=0$.

DEFINITION 4.1. The block diagonal matching field of size $3 \times n$ corresponding to a collection $\mathbf{a}=\left\{a_{1}, \ldots, a_{r}\right\}$ satisfying $\sum_{i=1}^{r} a_{i}=n$ is denoted $B \Lambda_{\mathbf{a}}$. This matching field is defined by:

(1) $B \Lambda_{\mathbf{a}}(I)=$ id if $\left|I \cap I_{s}\right| \geqslant 2$ where $s$ is the minimal $t$ such that $I_{t} \cap I \neq \varnothing$;

(2) $B \Lambda_{\mathbf{a}}(I)=(12)$ if $\left|I \cap I_{s}\right|=1$ where $s$ is the minimal $t$ such that $I_{t} \cap I \neq \varnothing$. A 2-block diagonal matching field is a block diagonal matching field with $r=2$.

EXAMPLE 4.2. Consider the case when $a_{1}=1$ and $a_{2}=n-1$. Then $I_{1}=\{1\}$ and $I_{2}=\{2, \ldots, n\}$. Then $B \Lambda_{1, n-1}(I)=$ id if and only if $I \subset I_{2}$. Otherwise, we have $1 \in I$ and 1 appears in the second row of column tableau corresponding to $I$. The matching field $B \Lambda_{1, n-1}$ is isomorphic to a pointed matching field $\Lambda$. This isomorphism is given by acting on $[n]$ by the transposition (12). In fact, the $\Lambda$-tableaux are then the PBW-tableaux from [9].

REMARK 4.3. Block diagonal matching fields can be generalised to size $k \times n$. The ideals of all 2-block diagonal matching fields are also quadratically generated. This can be proved in the same way as Theorem 1.4. However, we cannot prove analogues of Corollary 1.5 for Grassmannians $\operatorname{Gr}(k, n)$ for $k>3$ since in these cases quadratic generation of the initial ideals does not directly imply that the initial degeneration is toric.

In general there is a $\mathbb{Z}^{4}$-grading given by the number of elements of type $I_{1}$ in different rows of a tableau. A $3 \times d$ tableau $T$ is of degree $(\alpha, \beta, \gamma, d-\alpha-\beta-\gamma)$ where

(1) $\alpha=\mid\{$ Content of row 3 of $T\} \cap I_{1}|=|\left\{I \in T:\left|I \cap I_{1}\right|=3\right\} \mid$

(2) $\beta=\mid\{$ Content of row 1 of $T\} \cap I_{1}|-\alpha=|\left\{I \in T:\left|I \cap I_{1}\right|=2\right\} \mid$

(3) $\gamma=\mid\{$ Content of row 2 of $T\} \cap I_{1}|-\alpha-\beta=|\left\{I \in T:\left|I \cap I_{1}\right|=1\right\} \mid$

For two $\Lambda$-tableaux $T, T^{\prime}$ which are row-wise equal, these numbers are equal. This implies the following lemma.

Lemma 4.4. The ideal of a block diagonal matching field has a $\mathbb{Z}^{4}$-grading given by $(\alpha, \beta, \gamma, d-\alpha-\beta-\gamma)$ from above.

Proof of Theorem 1.4. Consider a binomial relation obtained from two $\Lambda$-tableaux $T$ and $T^{\prime}$ of size $3 \times d$ where $d>2$ whose contents are row-wise equal. By applying quadratic changes to the tableaux (changes involving only two columns) we will reduce the degree of this relation thus proving that the matching field ideal is quadratically generated.

Given a $\Lambda$-tableau $T$, arrange the columns so that the first columns are those for which the matching field assigns the identity permutation and to the last column the matching field assigns the transposition (12). Let $C$ denote the subtableau formed by the first columns and let $D$ denote the subtableau formed by the last columns. 
The tableaux $C$ and $D$ can each be put into semi-standard format. In other words, we can rearrange both $C$ and $D$ so that all rows are in weakly increasing order, and the columns of $C$ are strictly increasing, whereas the columns of $D$ are arranged so that the first and second entries are permuted from the diagonal order.

Now given a binomial relation obtained from two $\Lambda$-tableaux $T$ and $T^{\prime}$. We assume that $T$ (respectively $T^{\prime}$ ) is organized as a pair of subtableaux $C, D$ (respectively $\left.C^{\prime}, D^{\prime}\right)$ satisfying the requirements described above. If the first columns of $T$ and $T^{\prime}$ are equal, then we can cancel them from the binomial relation and it is not a minimal generator. We let $I$ and $I^{\prime}$ denote the first columns of $T$ and $T^{\prime}$, respectively. Otherwise by Lemma 4.4 the matching field relations are homogeneous with respect to the $\mathbb{Z}^{4}$-grading and so $\left|I \cap I_{1}\right|=\left|I^{\prime} \cap I_{1}\right|$

CASE 1. Suppose that $\left|I \cap I_{1}\right|=\left|I^{\prime} \cap I_{1}\right|=3$. In this case, the columns $I$ and $I^{\prime}$ could only differ in the second row. Suppose the entries of the second row of $I$ and $I^{\prime}$ are $j$ and $j^{\prime}$, respectively. We can also assume that $j<j^{\prime}$. Then there must be a $j$ in the second row of the tableaux $D^{\prime}$ since the contents are row-wise equal and $C^{\prime}$ is in weakly increasing order. Then swap the positions of $j$ and $j^{\prime}$ in the second row of $T^{\prime}$ so that the first columns of $T$ and $T^{\prime}$ now agree. Notice that we can exchange the position of $j$ with that of $j^{\prime}$ since $j, j^{\prime} \in I_{1}$ and $j^{\prime}$ was originally in the second row of a column whose the first and third entries were in $I_{2}$.

CAsE 2. Suppose that $\left|I \cap I_{1}\right|=\left|I^{\prime} \cap I_{1}\right|=2$. In this case, the columns $I$ and $I^{\prime}$ may only differ in the second and third rows but not in the first. Assume the column $I$ is $i, j, r$ and the column $I^{\prime}$ is $i, j^{\prime}, r^{\prime}$. If $j<j^{\prime}$ then just as above there must be a $j$ in the second row of $D^{\prime}$. We have that $j<r^{\prime}$ since $j$ is in the first block and $r^{\prime}$ is in the second block, so we can swap the positions of $j$ and $j^{\prime}$ in the second row of $T^{\prime}$.

Assume now that $j=j^{\prime}$, without loss of generality we can suppose that $r<r^{\prime}$. Then there is an $r$ in the third row of $D^{\prime}$. Suppose that the column containing $r$ is $s, t, r$. Then we can swap the positions of $r$ and $r^{\prime}$ since $t<r<r^{\prime}$ and we can place $r$ in the last row. Now the two first terms are equal and hence, the binomial is not a minimal generator.

CASE 3. Suppose that $\left|I \cap I_{1}\right|=\left|I^{\prime} \cap I_{1}\right|=1$. In this case, the tableaux $C$ and $C^{\prime}$ are empty. Then the first two columns must be equal since $T=D$ and $T^{\prime}=D^{\prime}$ and they are both in (transposed) semi-standard form.

CAse 4. Suppose that $\left|I \cap I_{1}\right|=\left|I^{\prime} \cap I_{1}\right|=0$. In this case, the entries of $I$ and $I^{\prime}$ can only differ in the first and third row. Suppose that the column $I$ is $r, s, t$ and that the column $I^{\prime}$ is $r^{\prime}, s, t^{\prime}$. Therefore $r, r^{\prime}<s<t, t^{\prime}$ and we can assume that $r^{\prime}<r$. Then there is a column in $T^{\prime}$ with $r$ in the first row and we can swap $r$ and $r^{\prime}$ in the first row of $T^{\prime}$. Thus we may assume that $r=r^{\prime}$ and without loss of generality that $t<t^{\prime}$. Then there must be a $t$ somewhere in the last row of $D^{\prime}$ and we can again swap $t$ and $t^{\prime}$ so that the columns are now equal. This completes the proof.

EXAMPLE 4.5. Here we provide examples of the manipulations of the matching field tableaux used throughout the proof of Theorem 1.4. The examples we consider are in $\operatorname{Gr}(3,8)$ with the block matching field given by $I_{1}=\{1,2,3,4\}$ and $I_{2}=\{5,6,7,8\}$.

The following is an example of a tableau in the semi-standard form as described in the beginning of the proof of Theorem 1.4,

$$
\begin{aligned}
& \begin{array}{lllll}
1 & 2 & 5 & 5 & 6
\end{array} \\
& \begin{array}{lllll}
3 & 3 & 6 & 2 & 4
\end{array} \\
& T=\begin{array}{lllll}
4 & 7 & 8 & 7 & 8
\end{array} . \\
& \underbrace{}_{C} \underbrace{8}_{D}
\end{aligned}
$$


The three columns on the left belong to $C$, since the entries are in ascending order and the two columns on the right belong to $D$. The degree of $T$ with respect to the grading described in Lemma $4.4(1,1,2,1)$ which can be seen by calculating $\alpha=|\{(1,3,4)\}|$, $\beta=|\{(2,3,7)\}|$ and $\gamma=\{(5,2,7),(6,4,8)\}$.

As an example of Case 1 in the proof, consider the tableaux

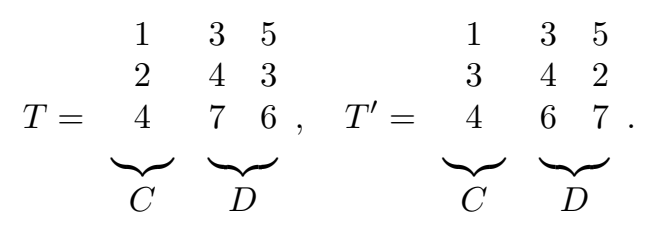

Then we may swap the entries 2 and 3 in the second row of $T^{\prime}$ to obtain a row-wise equal tableau whose left most column is $(1,2,4)$.

As an example of a tableau manipulation performed in Case 2 of the theorem consider the tableaux,

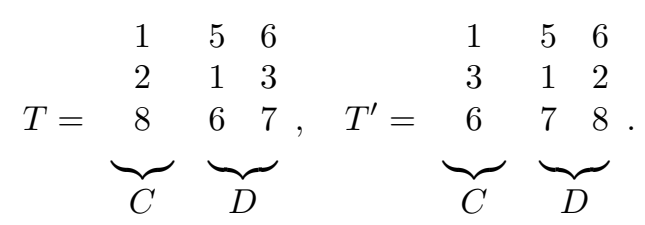

First we can swap entries 2 and 3 in the second row of $T^{\prime}$ to obtain tableau whose left most column is $(1,2,6)$. Then we swap entries 8 and 6 in the third row of $T$ to obtain a tableau whose left most column is also $(1,2,6)$. Note that we cannot swap entries 6 and 8 in the third row of $T^{\prime}$.

We omit examples of Case 3 in the proof since no swaps occur in this case. For an example of the manipulations made in Case 4, we consider instead the block matching field $I_{1}=\{1,2\}$ and $I_{2}=\{3,4,5,6,7,8\}$. Consider then the two tableaux,

$$
\begin{aligned}
& \begin{array}{llllllll}
4 & 5 & 3 & 6 & 3 & 6 & 4 & 5
\end{array} \\
& \begin{array}{llllllll}
5 & 7 & 1 & 2 & 5 & 7 & 1 & 2
\end{array} \\
& T=\begin{array}{llll}
6 & 8 & 5 & 7
\end{array}, \quad T^{\prime}=\begin{array}{lllll}
7 & 8 & 5 & 6
\end{array} . \\
& \underbrace{}_{C} \underbrace{r_{C}}_{D} \underbrace{2}_{D}
\end{aligned}
$$

First we swap entries 3 and 4 in the first row of $T^{\prime}$ to obtain a tableau whose left most column is $(4,5,7)$. Then we may swap entries 6 and 7 in the last row of $T^{\prime}$ to obtain a tableau whose left most column is $(4,5,6)$ which is identical to the left most column of $T$. Note that we can perform the swap of entries 3 and 4 in $T$ however we cannot swap entries 6 and 7 in $T$.

\section{Matching Field POLYTopes}

From a $k \times n$ matching field we can define a polytope in $\mathbb{R}^{n \times k}$. We expect these polytopes to be of interest in geometric combinatorics. Let $e_{i, j}$ denote coordinates on $\mathbb{R}^{n \times k}$. Given a matching field $\Lambda$, for each $I \in \mathbf{I}_{k, n}$ we set $v_{I, \Lambda}:=\sum_{i \in I} e_{i, \Lambda(I)(i)}$.

DEFINITION 5.1. Given a $k \times n$ matching field $\Lambda$ the matching field polytope $\Pi_{\Lambda}$ is the convex hull of the set of points $\left\{v_{I, \Lambda} \mid I \in \mathbf{I}_{k, n}\right\}$ in $\mathbb{R}^{n \times k}$.

Proposition 5.2. If $\Lambda$ is a coherent matching field then $\Pi_{\Lambda}$ is the polytope of the toric variety defined by the binomial ideal $\mathcal{I}_{\Lambda}$. 
Corollary 5.3. Let $\Lambda$ be a coherent $k \times n$ matching field then

$$
\frac{1}{[k(n-k)] !} \operatorname{vol}\left(\Pi_{\Lambda}\right) \leqslant \operatorname{deg} \operatorname{Gr}(k, n) .
$$

Recall that the degree of the Grassmannian is given by the number of standard Young tableaux of shape $\lambda=\left(\lambda_{1}, \ldots, \lambda_{k}\right)$ with $\lambda_{i}=n-k$ for all $i$. The number of standard Young tableaux is given by the hook-length formula

$$
\operatorname{deg} \operatorname{Gr}(k, n)=\frac{(k(n-k)) ! \prod_{1 \leqslant l \leqslant k-1} l !}{\prod_{1 \leqslant l \leqslant k}(n-l) !} .
$$

ExAMPLE 5.4. Let $\Lambda$ be the hexagonal matching field from Example 3.9. The matching field polytope $\Pi_{\Lambda}$ has Euclidean volume equal to $\frac{19}{181440}$ and normalised lattice volume equal to 38 . Whereas the polytope of the diagonal matching field from Example 3.8 has volume $\frac{1}{8640}$ and normalised lattice volume equal to 42 . The degree of the Grassmannian $(3,6)$ under the Plücker embedding is 42 . Table 1 lists all of the cones of the tropical Grassmannian coming from matching fields.

\begin{tabular}{|c||ccc|}
\hline Bounded 2-cell & Cone of $\operatorname{Gr}(3,6)$ & $f$-vector of $\Pi_{\Lambda}$ & $\mathbb{Z}$-vol \\
\hline \hline$\varnothing$ & EEEE & does not arise from a matching field & 42 \\
\hline Triangle & EEEG & $(20,123,386,728,882,700,358,111,18)$ & 42 \\
\hline Diagonal & EEFF(a) & $(20,122,372,670,766,571,276,83,14)$ & 42 \\
\hline Parallelogram & EEFF(b) & $(20,122,376,690,807,615,302,91,15)$ & 42 \\
\hline 4-gon & EEFG & $(20,122,378,701,832,645,322,98,16)$ & 42 \\
\hline Pentagon & EFFG & $(20,122,376,690,807,615,302,91,15)$ & 42 \\
\hline Hexagon & FFFGG & $(20,120,361,641,720,526,250,75,13)$ & 38 \\
\hline
\end{tabular}

TABLE 1. The $f$-vectors of the polytopes of the different possible initial degenerations of the Plücker embedding of the Grassmannian $\operatorname{Gr}(3,6)$. The description via the bounded 2-dimensional cell in the tropical hyperplane arrangement is from the classification in [11]. The first row is a toric degeneration which does not arise from a monomial degeneration of the Plücker forms, hence it does not come from a matching field.

Four of the matching fields in Table 1 arise as 2-block diagonal matching fields. Namely, the toric degeneration named "diagonal" comes from diagonal matching field (as well as the isomorphic block diagonal matching field $B \Lambda_{5,1}$ ). The toric degeneration named "parallelogram" comes from the block diagonal matching field $B \Lambda_{1,5}$. The "4-gon" comes from the matching field $B \Lambda_{4,2}$ and the "pentagon" comes from $B \Lambda_{2,4}$. The other rows do not arise from block diagonal matching fields.

REMARK 5.5. We say that two matching fields $\Lambda$ and $\Lambda^{\prime}$ are isomorphic if there exists an element $S_{k} \times S_{n}$ sending one to the other. In Table 1, the toric degenerations of $\operatorname{Gr}(3,6)$ from the tropical hyperplane arrangements with bounded cells a parallelogram and a pentagon produce isomorphic toric varieties. Already from the table we see that the corresponding polytopes have the same $f$-vector. However, it can be verified that the matching fields are not isomorphic. Therefore, the isomorphism type of the toric variety of a matching field does not determine the matching field. Also, the toric degeneration coming from the diagonal matching field is isomorphic to the one obtained from the non-isomorphic 2-block diagonal matching field $B \Lambda_{3,3}$. 
Acknowledgements. The authors are very grateful to Alex Fink, Jürgen Herzog, Mateusz Michałek, Felipe Rincón and Bernd Sturmfels for many helpful conversations. We would also like to thank Georg Loho, Volkmar Welker and two anonymous reviewers for helpful comments on a preliminary version of this manuscript.

We would like to remark that this paper was accepted for a poster in FPSAC 2018. However the extended abstract did not appear in the proceedings because the authors could not attend the conference.

\section{REFERENCES}

[1] Byung Hee An, Yunhyung Cho, and Jang Soo Kim, On the f-vectors of Gelfand-Tsetlin polytopes, Eur. J. Comb. 67 (2018), 61-77.

[2] David Bernstein and Andrei Zelevinsky, Combinatorics of maximal minors, J. Algebr. Comb. 2 (1993), no. 2, 111-121.

[3] Anders Björner, Michel Las Vergnas, Bernd Sturmfels, Neil White, and Günter M. Ziegler, Oriented matroids, second ed., Cambridge University Press, 1999.

[4] Lara Bossinger, Xin Fang, Ghislain Fourier, Milena Hering, and Martina Lanini, Toric degenerations of $\operatorname{Gr}(2, n)$ and $\operatorname{Gr}(3,6)$ via plabic graphs, Ann. Comb. 22 (2018), no. 3, 491-512.

[5] Lara Bossinger, Sara Lamboglia, Kalina Mincheva, and Fatemeh Mohammadi, Computing toric degenerations of flag varieties, in Combinatorial Algebraic Geometry, Springer-Verlag New York, 2017, pp. 247-281.

[6] Aldo Conca, Jürgen Herzog, and Giuseppe Valla, Sagbi bases with applications to blow-up algebras, J. Reine Angew. Math. 474 (1996), 113-138.

[7] Mike Develin and Bernd Sturmfels, Tropical convexity, Doc. Math. 9 (2004), 1-27.

[8] Anton Dochtermann and Fatemeh Mohammadi, Cellular resolutions from mapping cones, J. Comb. Theory, Ser. A 128 (2014), 180-206.

[9] Evgeny Feigin, $\mathbb{G}_{a}^{m}$ degeneration of flag varieties, Sel. Math., New Ser. 18 (2012), no. 3, 513-537.

[10] Alex Fink and Felipe Rincón, Stiefel tropical linear spaces, J. Comb. Theory, Ser. A 135 (2015), 291-331.

[11] Sven Herrmann, Anders Jensen, Michael Joswig, and Bernd Sturmfels, How to draw tropical planes, Electron. J. Comb. 16 (2009), no. 2, R6 (26 pages).

[12] Jürgen Herzog and Takayuki Hibi, Distributive lattices, bipartite graphs and Alexander duality, J. Algebr. Comb. 22 (2005), no. 3, 289-302.

[13] Takayuki Hibi, Every affine graded ring has a Hodge algebra structure, Rend. Semin. Mat., Univ. Politec. Torino 44 (1986), no. 2, 277-286.

[14] _ Distributive lattices, affine semigroup rings and algebras with straightening laws, Commutative Algebra and Combinatorics (Tokyo, Japan), Adv. Stud. Pure Math., vol. 11, Mathematical Society of Japan, 1987, pp. 93-109.

[15] Michael Kapovich and John J. Millson, The symplectic geometry of polygons in Euclidean space, J. Differ. Geom. 44 (1996), no. 3, 479-513.

[16] Kiumars Kaveh and Christopher Manon, Khovanskii bases, higher rank valuations and tropical geometry, arXiv preprint https://arxiv.org/abs/1610.00298, 2016.

[17] Mikhail Kogan and Ezra Miller, Toric degeneration of Schubert varieties and Gelfand-Tsetlin polytopes, Adv. Math. 193 (2005), no. 1, 1-17.

[18] Michał Lasoń and Mateusz Michałek, On the toric ideal of a matroid, Adv. Math. 259 (2014), $1-12$.

[19] Diane Maclagan and Bernd Sturmfels, Introduction to tropical geometry, Grad. Stud. Math., vol. 161, American Mathematical Society, Providence, RI, 2015.

[20] Ezra Miller and Bernd Sturmfels, Combinatorial commutative algebra, Grad. Texts Math., vol. 227, Springer-Verlag, New York, 2005.

[21] Takeo Nishinou, Yuichi Nohara, and Kazushi Ueda, Toric degenerations of Gelfand-Cetlin systems and potential functions, Adv. Math. 224 (2010), no. 2, 648-706.

[22] Hidefumi Ohsugi and Takayuki Hibi, Toric ideals generated by quadratic binomials, J. Algebra 218 (1999), no. 2, 509-527.

[23] Konstanze Rietsch and Lauren Williams, Newton-Okounkov bodies, cluster duality, and mirror symmetry for Grassmannians, arXiv preprint https://arxiv.org/abs/1712.00447, 2017.

[24] Lorenzo Robbiano and Moss Sweedler, Subalgebra bases, in Commutative Algebra, Lect. Notes Math., vol. 1430, Springer, Berlin, Heidelberg, 1990, pp. 61-87.

[25] David Speyer and Bernd Sturmfels, The tropical Grassmannian, Adv. Geom. 4 (2004), no. 3, 389-411. 
[26] Bernd Sturmfels, Gröbner bases and convex polytopes, Univ. Lect. Ser., vol. 8, American Mathematical Society, 1996.

[27] Bernd Sturmfels and Andrei Zelevinsky, Maximal minors and their leading terms, Adv. Math. 98 (1993), no. 1, 65-112.

[28] Neil L. White, A unique exchange property for bases, Linear Algebra Appl. 31 (1980), 81-91.

[29] Jakub Witaszek, The degeneration of the Grassmannian into a toric variety and the calculation of the eigenspaces of a torus action, J. Algebr. Stat. 6 (2015), no. 1, 62-79.

Fatemen Mohammadi, University of Bristol, Bristol, BS8 1TW, UK

E-mail : fatemeh.mohammadi@bristol.ac.uk

Kristin Shaw, University of Oslo, P.O. box 1053, Blindern, 0316 OSLO, Norway

E-mail : krisshaw@math.uio.no 